\title{
Horse sense: social status of horses (Equus caballus) affects their likelihood of copying other horses' behavior
}

Konstanze Krueger, Jürgen Heinze

corresponding author: Konstanze Krueger: konstanze-krueger@equine-science.de

'The original publication is available at www.springerlink.com' December 2007

Animal Cognition Volume 11, Number 3, 431-439, DOI: 10.1007/s10071-007-0133-0

\begin{abstract}
Animals that live in stable social groups need to gather information on their own relative position in the group's social hierarchy, by either directly threatening or by challenging others, or indirectly and in a less perilous manner, by observing interactions among others. Indirect inference of dominance relationships has previously been reported from primates, rats, birds, and fish. Here, we show that domestic horses, Equus caballus, are similarly capable of social cognition. Taking advantage of a specific "following behavior" that horses show towards humans in a riding arena, we investigated whether bystander horses adjust their response to an experimenter according to the observed interaction and their own dominance relationship with the horse whose reaction to the experimenter they had observed before. Horses copied the "following behavior"

towards an experimenter after watching a dominant horse following but did not follow after observing a subordinate horse or a horse from another social group doing so. The "following behavior," which horses show towards an experimenter, therefore appears to be affected by the demonstrator's behavior and social status relative to the observer.
\end{abstract}

\section{Keywords}

\section{Copying Horse Social cognition Sociality}

\section{Introduction}

Recognizing individuals, tracking their social status, and inferring relationships among group members are beneficial for animals that live in groups. Several studies document that primates, birds, and fish can indirectly draw conclusions about their own relative dominance status by observing interactions among others (Grosnick et al. 2007; Ame' et al. 2006; Robert et al. 2003; Cheney and Seyfarth 1990). For example, pinyon jays, Gymnorhinus cyanocephalus, behave more submissively to an unfamiliar bird after previously observing it winning a contest with a familiar, dominant bird than to a control bird (Paz-y Miño et al. 2004). Moreover, it has been reported that sociality affects the tendency of copying the mate choice of observed conspecifics in birds (Galef et al. 1998; White 2004) and fish (Dugatkin and Godin 1992; Goldschmidt et al. 1993; Dugatkin 2007; for review see Kraak 1996). Horses are social animals, which live in herds structured by stable dominance hierarchies (Goldschmidt-Rothschild and Tschanz 1978; Houpt et al. 1978). Much has been speculated about the skills of horses, such as the feigned arithmetic proficiency of "Clever Hans." Nevertheless, their social cognitive abilities, i.e., the processing, encoding, storage, retrieval, and application of social information, have only rarely been investigated under controlled conditions or in the field, and previous research has led to ambiguous and controversially discussed results (reviewed by Murphy and Arkins 2007; Nicol 2002). For example, Baer et al. (1983), Baker and Crawford (1986), and Clarke et al. (1996) investigated how horses perform in a discrimination task, in which observer horses were allowed to watch a trained demonstrator horse feeding only from one of two differently colored buckets and to search for food in the buckets after removal of the demonstrator. From the outcome of this test it was concluded that horses avoid the feeding territory of the demonstrator horse, and that watching a feeding demonstrator significantly shortened the latency until the observer approached the area.

The authors suggested that horses learn "something" about the general location of food from the demonstrator's performance. In her review on equine learning, Nicol (2002) therefore noted an urgent need for studies on social learning and social cognition in horses, as facts and presumptions in previous studies are often difficult to separate. To understand how horses communicate and how their herds are structured is also of fundamental importance for horse 
training (Feist and McCullough 1976; GoldschmidtRothschild and Tschanz 1978; Houpt et al. 1978; Feh 2002; McDonnell 2003).

What animals know about themselves and their social environment (McGregor and Dabelsteen 1976; Oliveira et al. 1998) has recently been studied by observing the reaction of uninvolved bystanders to dyadic encounters among group members. In an analogy of this approach we took advantage of the "following behavior" horses show when confronted by an experimenter in the "round pen technique", a method for the initial training of horses, which has been established by "horse-whisperers" (Rivera et al. 2002; Sighieri et al. 2003; Miller and Lamb 2005). Horses behave in a standardized way when approached and chased by an experimenter in the riding arena. They first run around the experimenter in circles, turn an ear to her, start licking and chewing, and finally follow the experimenter when she turns her back and slowly moves away. Horses begin to follow sooner and sooner with each repetition of this experiment until they immediately follow the experimenter and also unfamiliar persons (Krueger 2007). The behavior is limited to the surroundings in which the horses have first followed the experimenter, and they do not show this behavior when taken to a pasture. This suggests that horses learn to follow in this particular situation and also show some generalization (Krueger 2007).

For the present study we investigated whether domestic horses (bystanders) observe follow-up interactions between another horse (demonstrator) and an experimenter. Assuming that horses memorize information about the social structure of their herd and that they draw conclusions from observed interactions among herd members, we expected that bystander horses would adjust their own reaction in a similar situation according to the behavior of the demonstrator and its relative social status.

\section{Methods}

Animals

We investigated the behavior of 38 riding horses (composed of 26 mares and 12 geldings), which were 33 European bred Warmblood horses, two Draft-horses, two Thoroughbreds and one pony crossbreed, all aged between 3 and 22 years. Horses were individually identified by their brands and coloration. For completeness, two horses, which did not participate in the tests because of bad health, are included in the hierarchies in the appendix but labeled with an asterisk. The horses were kept in different conditions before and during experiments (four larger groups with $11,6,9$, and 4 horses, 3 pairs, and 4 solitary horses). Group one was established 66 months, group two 9 months, group three 96 months, group four 61 months, group five 34 months, group six 13 months, and group seven 18 months prior to the test. Most horses were housed in the same stable, in individual boxes sized $3 \mathrm{~m} 94 \mathrm{~m}$ with a bedding of wood shavings overnight and in social groups on pastures during the day, or constantly in social groups in open stables (social group 2 with six horses). Only social group 3 with nine horses was kept and tested in a different riding stable, and housed overnight in $3 \mathrm{~m} 94$ $m$ boxes on straw bedding. Horses were fed with hay twice a day and with a granola-cereal mixture three times a day. In addition they fed on the grass on their pastures.

None of the horses had participated in such an experiment before. Several horses had experience with the "round pen technique", while others were considered to be "naý"ve" in this respect, though we do not have a complete record of their training history (for detailed information see Tables 1, 2, 3, 4). Fourteen horses took part in the experiment both as demonstrator and bystander, eight horses served exclusively as demonstrator, and 15 horses exclusively as bystander (Tables 1, 2, 3, 4). To avoid a possible impact of using individuals repeatedly in the tests, we performed a second analysis of the data excluding all bystanders that had previously been used as demonstrators, participated a third time, or had a dominance score similar to that of their demonstrator (Wanda-Heaven). All bystanders participating in test 2 had previously also been bystander in test 1 , because we aimed at investigating whether they modify their behavior in accordance with that of changed demonstrator behavior. Six particularly welltrained demonstrator horses participated up to four times in a single test. The maximum performing time matched their normal daily workout time.

\section{Dominance relationships}

Before starting the experiments, we determined the dominance relationships among the horses in the field by observing agonistic encounters, such as ap-

Table 1 General Estimating Equations (GEE) for possible effects of various parameters on the following behavior of bystanders

\begin{tabular}{|l|l|l|l|l|}
\hline & \multicolumn{2}{|l|}{ GEE demonstrator } & \multicolumn{2}{l|}{ GEE bystander } \\
\hline & Robust $z$ & $P$ & Robust $z$ & $P$ \\
\hline Intercept & -0.724 & 0.767 & -0.103 & 0.541 \\
\hline Size & 0.779 & 0.782 & -0.043 & 0.517 \\
\hline Age & 1.677 & 0.953 & 0.106 & 0.542 \\
\hline Gender & -1.492 & 0.932 & -0.958 & 0.831 \\
\hline $\begin{array}{l}\text { D u r a t i o n } \\
\text { chased }\end{array}$ & -1.601 & 0.945 & 1.487 & 0.931 \\
\hline Experienced-naive & & & \\
\hline $\begin{array}{l}\text { Duration in } \\
\text { group }\end{array}$ & -0.187 & 0.574 & 0.237 & 0.594 \\
\hline
\end{tabular}

None of these parameters has a significant affects on the following behavior (for details see text) 


\begin{tabular}{|c|c|c|c|}
\hline Demonstrator & Following grade & Bystander & Following grade \\
\hline Momo & 2 & Demi• & 1 \\
\hline Massimo & 0 & Komet $^{*}$ & 0 \\
\hline Francis & 0 & Traum* & 0 \\
\hline Gero & 0 & Filou* $^{*}$ & 0 \\
\hline Heaven & 1 & Wanda* & 1 \\
\hline Heaven & 0 & Lise• & 0 \\
\hline Heaven & 0 & Selina• & 1 \\
\hline La Luna & 0 & Tantieme• & 0 \\
\hline Amigo & 3 & Pina* & 3 \\
\hline Demi & 1 & Gigolo• & 1 \\
\hline Elan & 3 & Lara* & 3 \\
\hline Pan Tau & 3 & Mary* & 2 \\
\hline
\end{tabular}

\begin{tabular}{|c|c|c|c|}
\hline Demonstrator & Following grade & Bystander & Following grade \\
\hline Francis & & Gigolo & 3 \\
\hline Gero & 3 & Filou & 3 \\
\hline La Luna & 3 & Tantieme & 3 \\
\hline Massimo & 3 & Komet & 3 \\
\hline Francis & 3 & Traum & 3 \\
\hline Traum & 3 & Mary & 2 \\
\hline Colleen & 2 & Lise & 3 \\
\hline Pan Tau & 3 & Demi & 1 \\
\hline Colleen & & Selina & 2 \\
\hline
\end{tabular}

proaches, retreats, threats to bite or kick, bites, kicks, and chases (Feist and Mc Cullough 1976; Rivera et al. 2002; McDonnell 2003). For specific sampling of the dominance interactions described above horses were observed over one hour each on two separate days (pre-test observation time: 2 hours per group or pair). Observations were evenly distributed over day light period. To corroborate our results, we observed dominance relationships twice again in the same manner, immediately after and within two weeks after the tests (post-test observation time: $2 \times 2$ hours, total observation time: 6 hours per group or pair). Interactions among the horses were recorded continuously. Subsequently, dominance scores of individuals were calculated by adding instances of active antagonism and subtracting cases of retreat (table 6).

\section{Experimental procedure}

In October 2005 we conducted four different tests on five subsequent days in two different stables, which were located in Obertraubling and Beratzhausen, both admin. district Regensburg, Germany. Tests 1, 2 , and a part of test 3 were performed in stable one on three respective days between 9:00 a.m. and 3:00 p.m. The remaining tests 3 were performed on the fourth day in stable two between 8:00 a.m. and 12:00

Table 4 "Following grades" test 3: dominant bystanders do not copy the behavior of subordinate demonstrators

\begin{tabular}{|l|l|l|l|}
\hline Wanda & 3 & Heaven* & 0 \\
\hline Traum & 3 & Francis $\bullet$ & 3 \\
\hline Sylvester & 3 & Candy $\bullet$ & 0 \\
\hline Traum & 3 & Indra $\bullet$ & 0 \\
\hline Traum & 3 & Momo $\bullet$ & 0 \\
\hline Nivera & 3 & Rosella* & 0 \\
\hline Mary & 3 & Pan Tau $\bullet$ & 1 \\
\hline Komet & 3 & Massimo $\bullet$ & 0 \\
\hline Gloria & 1 & Nircade* & 1 \\
\hline Nicki & 3 & Mon Cheri* & 0 \\
\hline Ronda & 3 & Nivera* & 0 \\
\hline Nicki & 3 & Walgadena* & 0 \\
\hline Nicki & 3 & Ronie* & 0 \\
\hline Ronda & 3 & Nicki* & 0 \\
\hline * bystander naïve, $\bullet$ bystander familiar with "round pen training \\
\hline
\end{tabular}

a.m. On the fifth day we returned to the first stable and tested the horses for test 4 between 10:00 a.m. and 3:00 p.m.

For the specific tests we hypothesized that:

Test 1: subordinate bystanders copy the behavior of dominant demonstrators, i.e., they follow if they observe the demonstrator following, and they do not follow if the demonstrator does not follow. We paired higher-ranking demonstrators with lower-ranking bystanders ( $n=12,7$ bystanders experienced with "round pen training", 5 naïve).

Test 2: those bystanders, which had not followed in test 1 after seeing a dominant demonstrator not following, change their behavior and follow in a second experiment after seeing another dominant demonstrator following. We paired bystanders, which had not followed (following grades 0 or 1 ) in test 1 with demonstrators which followed (following grades 2 or 3 ) ( $n=9$, all experienced with "round pen training").

Test 3: dominant bystanders do not copy the behavior of subordinate demonstrators.

We paired lower-ranking demonstrators with higherranking bystanders ( $n=14,7$ bystanders experienced with "round pen training", 7 naïve).

Test 4: bystander horses do not copy the behavior of unfamiliar demonstrator horses.

We paired bystanders with demonstrators from different social groups ( $\mathrm{n}=8,5$ bystanders experienced with "round pen training", 3 naïve). Half of the demonstrator horses were of high rank and the other half of low rank in their respective groups.

All experiments were conducted in either of the two respective riding arenas $(20 \mathrm{~m} \times 50 \mathrm{~m})$ attached to the stables were the horses were kept, which were divided into $20 \mathrm{~m} \times 20 \mathrm{~m}$ test areas and $20 \mathrm{~m} \times 30$ 
m observation areas by a rope (Fig. 1). The respective tests horses, which were chosen as "bystander" according to its social status and affiliation, were given the opportunity to explore the test area for $2-5$ min., depending on the time they needed to adjust to the test situation. Thereafter, the bystander was tested whether it would spontaneously follow the experimenter. The experimenter tested each bystander horse for 5 minutes, in which she first walked towards the horse, clapped on its shoulder, walked away from it while giving it the opportunity to follow, and, if it did not follow, continued to walk in close proximity to the horse, waiting for the horse to follow. Four horses (Elan, Sylvester, Colleen and Ronda) followed immediately and were not used as bystanders but as demonstrators in subsequent tests. Horses that did not follow were released in the observation area of the riding arena, where they were allowed to move freely. A demonstrator horse was led into the test area and given a corresponding amount of time to adapt to the new situation and to explore the arena, but was not allowed to approach the bystander. Subsequently, the bystander had the opportunity to observe an interaction between the experimenter and the demonstrator horse in the test area (Fig. 1). The behavior shown by the horses in the "round pen training" has shortly been outlined in the introduction, and a detailed description has been published elsewhere (Krueger 2007). In short, the "round pen training" can be described as an interaction between horses and humans in which the experimenter first gives the horse the opportunity to follow her spontaneously. In case it does not follow it will be chased away so that it gallops in circles around the experimenter. The experimenter repeatedly stops chasing, walks to the horse, claps its shoulder, and gives it the opportunity to follow until it finally does so.

In our experiments all demonstrator horses, even those, which followed the experimenter spontaneously, were chased in the test area for at least $2 \mathrm{mi}-$ nutes, but not longer than 15 minutes. According to a previous study (Krueger 2007), most, though not all,

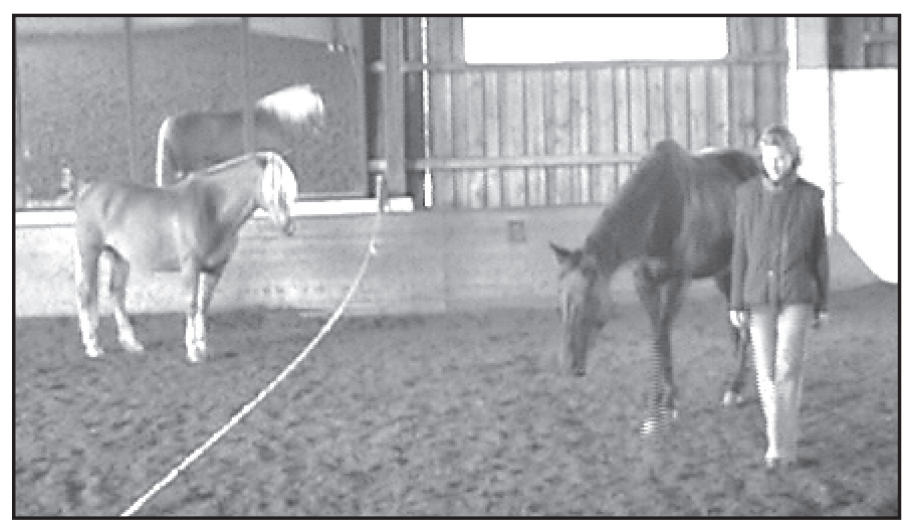

Fig. 1 Experimental set-up used in the bystander test. A bystander horse watches a demonstrator horse following an experimenter horses follow an experimenter after being chased for this time span. The time horses needed to be chased before following the experimenter decreased significantly with every round of training (Krueger 2007). In test 2, we took advantage of the experience that demonstrators, which did not follow on the first day of "round pen training" within 15 minutes, often would do so in the following training round. If the demonstrator horse followed the experimenter, both continued to walk across the test arena for 15 minutes. Demonstrator horses that did not follow after chasing were given the opportunity to follow the experimenter for 15 minutes to standardize the interaction time a bystander could observe.

Immediately after the interaction between the demonstrator horse and the experimenter we investigated again whether the bystander horse would now spontaneously follow the experimenter. The experimenter walked to the bystander horse in the observation area and gave it the opportunity to follow, as she had done before the interaction with the demonstrator horse, but now for 15 minutes. In case the horse followed the experimenter spontaneously, they kept on walking through the observation area for $15 \mathrm{mi}-$ nutes. If the horse did not follow, the experimenter continued to walk in close proximity to the horse for 15 minutes.

\section{Experimenter}

Five experimenters took part in the study: experimenter 1 in tests 1 and 2, experimenters 2 and 3 in test 3 , and experimenter 4 in test 4 . The fifth experimenter observed and continuously videotaped the interactions from outside the riding arena. In previous studies, horses had shown similar reactions to the different experimenters, suggesting that the different outcomes in the various tests did not depend on variation in the personality, behavior, or posture of the respective experimenters (Krueger 2007). All experimenters, with exception of the fifth experimenter outside of the riding arena, were unfamiliar to the horses. In addition, the experiments were conducted blindly, i.e., the experimenters neither knew the dominance status of the horses investigated nor the predicted outcome of the tests, as test pairs were chosen by another person, who was familiar with the horses and knew how the horses had to be paired for the experiment. In addition to observing and recording all behavior, the experiments were continuously videotaped for further analyses and conservation of the data.

\section{Categories and Statistics}

The "following behavior" of the horses was categorized as:

Grade 0: Horse does not follow the experimenter within 15 minutes

Grade 1: Horse follows within 5 sec. after being ap- 
proached by the experimenter but only for a maximum of 2 minutes

Grade 2: Horse follows immediately, stays within 2 to $3 \mathrm{~m}$ behind the shoulder of the experimenter and follows for $15 \mathrm{~min}$, but interrupted by periods of notfollowing of up to 1 minute

Grade 3: Horse follows immediately, stays within $1 \mathrm{~m}$ to $2 \mathrm{~m}$ behind the shoulder of the experimenter, and continuously follows for 15 minutes in any direction. Grades 0 and 1 were considered as "not following", grades 2 and 3 were considered as "following".

For statistical analysis we used the R-Project statistical environment (2007). Potential influences of different age, sex, size, duration of residency in the social group, duration of the chasing time of demonstrators, and previous experience of bystanders in "round pen training" on the following behavior of bystanders were investigated with Generalized Estimating Equations (GEEs), which are methods for parameter estimation for correlated data (Liang and Zeger 1986). We plotted one GEE to analyze the potential effects of these factors on the demonstrators and another for potential effects of traits on the bystanders. Because the different social groups were first established in different years, we calculated an individual horse's "duration of residency in the group" by dividing the individual's duration of residency by the total duration of their respective group.

The change in the behavior of bystander horses tested twice in tests 1 and 2 was investigated using a Wilcoxon rank sum test. Fisher's exact test is robust with small sample sizes, ordinal data and ties, and was thus used to compare the probability of following grades of bystanders matching those of demonstrator horses in tests 1, 3, and 4 .

\section{Results}

The behavior of bystander horses allowed to watch the interaction between an experimenter and a demonstrator horse differed enormously. Neither age, sex, size, duration of residency in the social group, duration of the chasing time of demonstrators, nor experience of bystanders in "round pen training" had a significant effect on bystander behavior (all $p>$ 0.05 , see table 1 ).

After observing a "round pen" interaction between an experimenter and a demonstrator horse, subordinate bystander horses usually copied the behavior of familiar, dominant demonstrators. They significantly followed after observing a familiar, dominant demonstrator doing so, but they did not follow after the demonstrator had not followed (Fisher's Exact test: $\mathrm{N}=12, \mathrm{p}=0.003$, Fig. 2, Table 2). Using the reduced data set, i.e., excluding all those bystanders that had also performed as demonstrators, participated repeatedly in tests 1,3 , or 4 , or whose dominance re-

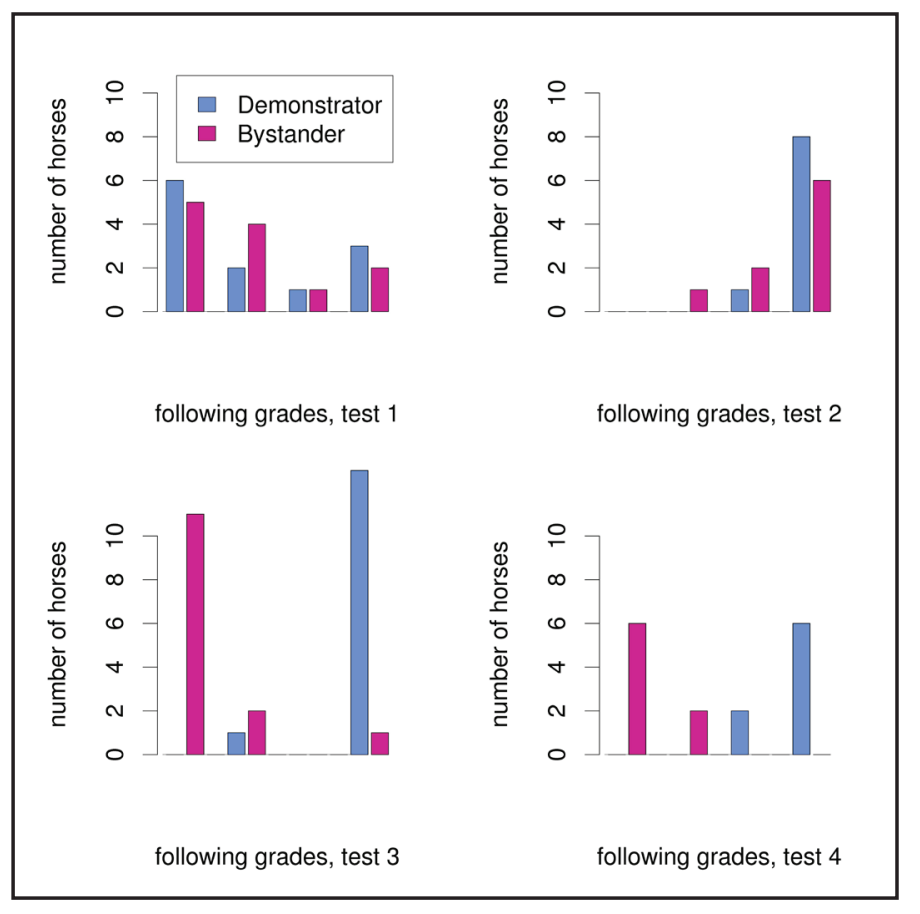

Fig. 2 "Following behavior" observed during the experiments. In test 1 , subordinate bystanders copied the behavior of a dominant demonstrator, i.e., they followed the experimenter after having seen a dominant demonstrator doing so but did not follow if the demonstrator had not followed $(n=12)$. In test 2 , bystanders that had not followed in test 1 now followed after seeing a dominant demonstrator doing so ( $\mathrm{n}=$ 9 ). In test 3 , dominant bystanders did not copy the behavior of subordinate demonstrators $(n=14)$, and in test 4 , bystander horses did not copy the behavior of unfamiliar demonstrator horses regardless of their social status $(n=8)$. Grades 0 and 1 were considered as "not following", Grades 2 and 3 were considered as "following" (see text for details)

lationships to the demonstrator were unclear, gave a similar result (Fisher's Exact test: $\mathrm{N}=11, \mathrm{p}=0.010$ ). In test 2, those bystanders, which had not followed in test 1 after seeing a dominant horse not following, followed after seeing a dominant demonstrator doing so in this second experiment. Thus, subordinate horses again copied the behavior of dominant demonstrators and significantly changed their behavior compared to test 1 (bystander performance in tests 1 vs. test 2 , Wilcoxon rank sum test: $N=9, W=3.5, p$ $=0.001$; reduced data set: $N=8, W=3.5, p=0.002$; Fig. 2, Table 3).

In contrast, the results of test 3 show that dominant bystander horses do not copy the behavior of subordinate demonstrator horses (Fisher's Exact Test: $\mathrm{N}=$ 14, $p=0.210$, Table 4, Fig. 2; reduced data set: $N=$ $7, p=0.143)$.

Similarly, in test 4 bystander horses did not copy the behavior of unfamiliar demonstrator horses (Fisher's Exact Test: $\mathrm{N}=8, \mathrm{p}=0.460$, Table 5, Fig. 2). The sample size in the reduced data set was too small ( $N$ $=3$ ) for doing a meaningful statistical test $(p=1.000)$. 


\section{Discussion}

Our study suggests that domestic horses are not only capable of distinguishing between familiar and unfamiliar horses but also of recognizing the social status of familiar horses relative to their own. Furthermore, they extract information from observing interactions between a familiar horse and a human experimenter and adjust their behavior with respect to the observed horse's reaction and relative dominance status when later confronted with the experimenter in a similar situation.

The data clearly document that the behavior of subordinate horses matches that of the observed, dominant demonstrator horses: they were reluctant to follow the experimenter when the observed dominant horses had not followed, but followed after the observed dominant horses had done so. In contrast, dominant bystanders did not adjust their behavior to the experimenter after observing a subordinate or an unfamiliar demonstrator. Instead, even dominant horses that had followed as demonstrators did not follow after observing a subordinate horse perfectly following the experimenter. This also shows that, though horses learn to follow the experimenter in repeated tests (Krueger 2007), their behavior as bystanders is not affected by their prior experience in the "round-pen technique". This is also corroborated by the lack of an influence of "previous experience" as a confounding variable in the analysis and by the results from a reduced data set which excluded horses that had repeatedly taken part in the experiment. An impact of learning in repeated tests therefore was excluded. Furthermore, neither age, sex, size, duration of residency in the social group, nor duration of the chasing time of demonstrators had a significant effect on bystander behavior.

Instead, horses appear to be indeed capable of social cognition, a competence which is beneficial for life in social groups but has previously been reported almost exclusively from primates and highly social birds (Treichler and Van Tilburg 1996; Roberts et al. 2003; Acuna et al. 2002; Paz-y Miño et al. 2004; Allen 2006; Moses et al. 2006). Observing another horse's behavior in a social interaction and drawing conclusions on its social status might be skills that evolved because they facilitate the integration of individuals into alien social groups, which is a common phenomenon among free ranging horses at the age of three to five years (Tyler 1972; Berger 1986). Such integration would be much more costly if horses would have to test the social status of each group member individually.

The formation of alliances among free-ranging stallions (Feh 1999) and the use of human-given cues in object choice tests, albeit limited (McKinley 2000), had previously suggested the occurrence of cognitive abilities in horses. However, studies on cognition in relation to food could not document social learning in horses (Baer et al. 1983; Baker and Crawford 1986; Clarke et al. 1996; Lindberg et al. 1999). Nevertheless, Baker and Crawford (1986) and Clarke et al. (1996) concluded that sociality might affect the feeding choice of bystander horses in that they avoid the feeding areas of demonstrator horses. A similar influence of social rank and social affiliation on feeding preferences has been shown in several other species, including primates and chicken (Fragaszy and Visalberghi 1996; Nicol and Pope 1999; Nicol 2006). Similarly, sociality is known to influence mate choice copying in vertebrates (Dugatkin 2007; Godin et al. 2005; White 2004; Dugatkin and Godin 1993). However, both in mate choice copying in vertebrates (White 2004) and our study, it appears to be difficult to distinguish social learning from other social effects, such as social facilitation. The following of bystanders after observing a familiar, dominant demonstrator horse following the experimenter might simply result from dominants behaving in a more conspicuous way, and bystanders may have learnt during previous interactions that it pays to observe conspicuous individuals in order to avoid being threatened or displaced. This might explain the copying behavior without requiring complex cognitive skills. However, neither size nor age of the demonstrator had a significant impact on the following behavior of bystander horses. Moreover, bystanders only copied the behavior of familiar dominants, suggesting that they did not simply react to some general dominance features. To clarify whether social learning is involved in horses, it might be helpful to analyze the degree of attention bystander horses pay to the interaction of a demonstrator horse especially in reference to the horses' social ranks and affiliation to social groups. In addition, experiments should be conducted to gain more information about whether horses generalize and memorize the observed following response to a human experimenter.

\section{Acknowledgements}

We thank Bernd Kramer, Beat Tschanz, Fritz Trillmich, Katherine Houpt, Norbert Sachser, and Michael Tomasello for helpful discussion, as well as Elisabeth $\mathrm{D}$ 'Antoni for language correction and the horse owners for providing us with their horses. The study was supported by an HWP II grant of the University of Regensburg. We declare that the experiments comply with the current laws of the country in which they were performed. 


\section{References}

Acuna BD, Sanes JN, Donoghue JP (2002) Cognitive mechanisms of transitive inference. Exp Brain Res doi 10.1007/s00221-002-1092-y

Allen C (2006) Transitive inference in animals: Reasoning or conditioned associations? In: Hurley S, Nudds M (eds) Rational Animals? Oxford University Press, Oxford, pp 175-186.

Amé J-M, Halloy J, Rivault C, Detrain C, Deneubourg JL (2006) Collegial decision making based on social amplification leads to optimal group formation. Proc Natl Acad Sci U S A doi 10.1073/ pnas.0507877103

Baer KL, Potter GD, Friend TH, Beaver BV (1983) Observation effects on learning in horses. Appl Animal Ethol doi 10.1016/0304-3762(83)90121-9

Baker AEM, Crawford BH (1986) Observational learning in horses. Appl Anim Behav Sci doi 10.1016/0168-1591(86)90017-1

Berger J (1986) Wild Horses of the Great Basin: Social Competition and Population Size. University of Chicago Press, Chicago.

Cheney D.L., Seyfarth RM (1990) How monkeys see the world: Inside the mind of another species. University of Chicago Press, Chicago.

Clarke JV, Nicol CJ, Jones R, McGreevy PD (1996) Effects of observational learning on food selection in horses. Appl Anim Behav Sci doi 10.1016/01681591(96)01071-4

Dugatkin LA. (2007) Developmental environment, cultural transmission, and mate choice copying. Am J Primatol doi 10.1007/s00114-007-0238-y

Dugatkin LA, Godin J-GJ (1993) Female mate copying in the guppy (Poecilia reticulata): age-dependent effects. Behav Ecol 4, 289-292

Dugatkin LA, Godin JG (1992) Reversal of female mate choice by copying in the guppy (Poecilia reticulata). Proc Biol Sci doi 10.1098/rspb.1992.0101

Feh C (1999) Alliances and reproductive success in Camargue stallions. Anim Behav doi 10.1006/ anbe.1998.1009

Feh C (2002) Relationships and Communication in Socially Natural Horse Herds.

Feist JD, McCullough DR (1976) Behavior patterns and communication in feral horses. Z Tierpsychol 41, 337-371

Fragaszy D, Visalberghi E (1996) Primates "primacy" reconsidered. In: Heyes C, Galef BG (eds) Social learning in animals: the roots of culture. Academic Press, Inc., pp 65-84.

Galef J, Bennett G., White DJ (1998) Mate-choice copying in Japanese quail, Coturnix coturnix japonica. Anim Behav 55, 545-552
Godin J-GJ, Herdman EJE, Dugatkin LA (2005) Social influences on female mate choice in the guppy, Poecilia reticulata: generalized and repeatable trait-copying behaviour. Anim Behav 69, 999-1005 Goldschmidt T, Bakker TCM, Feuth-de Bruijn E (1993) Selective copying in mate choice of female sticklebacks. Anim Behav 45, 541-547

Goldschmidt-Rothschild VB, Tschanz B (1978) Soziale Organisation und Verhalten einer Jungtierherde beim Camargue-Pferd. Z Tierpsychol 46, 372-400

Grosenick L, Clement TS, Fernald RD (2007) Fish can infer social rank by observation alone. Nature doi 10.1038/nature05511

Houpt KA, Law K, Martinisi V (1978) Dominance hierarchies in domestic horses. Appl Animal Ethol doi 10.1016/0304-3762(78)90117-7

Kraak SBM (1996) 'Copying mate choice: Which phenomena deserve this term? Behav Process doi 10.1016/0376-6357(95)00020-8

Krueger K (2007) Behaviour of horses in the "round pen technique”. Appl Anim Behav Sci doi 10.1016/j. applanim.2006.04.021

Liang K-Y, Zeger SL (1986) Longitudinal data analysis using generalized linear models. Biometrika73, 13-22

Lindberg AC, Kelland A, Nicol CJ (1999) Effects of observational learning on acquisition of an operant response in horses. Appl Anim Behav Sci doi 10.1016/S0168-1591(98)00184-1

McDonnell SM (2003) The Equid Ethogram: A Practical Field Guide to Horse Behavior. Eclipse Press, Lexington, Kentucky.

McGregor PK, Dabelsteen T (1976) Communication Networks. In: Kroodsma DE, Miller EH (eds) Ecology and evolution of acoustic communication in birds. Cornell University Press, Ithaca, pp 409425.

McKinley J, Sambrook TD (2000) Use of human-given cues by domestic dogs (Canis familiaris) and horses (Equus caballus). Anim Cogn doi 10.1007/ s100710050046

Miller R, Lamb R (2005) The Revolution in Horsemanship: And What It Means to Mankind. The Lyons Press, Guilford,Connecticut.

Moses SN, Villate C, Ryan JD (2006) An investigation of learning strategy supporting transitive inference performance in humans compared to other species. Neuropsychologia doi 10.1016/j.neuropsychologia.2006.01.004

Murphy J, Arkins S (2007) Equine learning behaviour. Behav Process doi doi:10.1016/j.beproc.2006.06.009

Nicol CJ (2002) Equine learning: progress and suggestions for future research. Appl Anim Behav Sci doi 10.1016/S0168-1591(02)00093-X 
Nicol CJ (2006) How animals learn from each other. Appl Anim Behav Sci doi 10.1016/j.applanim.2006.04.004

Nicol CJ, Pope SJ (1999) The effects of demonstrator social status and prior foraging success on social learning in laying hens. Anim Behav doi 10.1006/ anbe.1998.0920

Oliveira RF, McGregor PK, Latruffe C (1998) Know thine enemy: fighting fish gather information from observing conspecific interactions. Proceedings of the Royal Society B: Biological Sciences doi 10.1098/rspb.1998.0397

Paz-Y-Mino C. G., Bond AB, Kamil AC, Balda RP (2004) Pinyon jays use transitive inference to predict social dominance. Nature doi 10.1038/nature 02723

R Development Core Team (2007) R: A Language and Environment for Statistical Computing. Foundation for Statistical Computing, Vienna.

Rivera E, Benjamin S, Nielsen B, Shelle J, Zanella AJ (2002) Behavioral and physiological responses of horses to initial training: the comparison between pastured versus stalled horses. Appl Anim Behav Sci doi 10.1016/S0168-1591(02)00091-6

Robert M, Seyfarth RM, Cheney DL (2003) The Structure of Social Knowledge in Monkeys. In: de Waal T (ed) Animal Social Complexity: Intelligence, Culture, and Individualized Societies. Harvard University Press, Cambridge, Massachusetts.

Sighieri C, Tedeschi D, De Andreis C, Petri L, Baragli $P$ (2003) Behaviour patterns of horses can be used to establish a dominantsubordinate relationship between man and horse. Animal Welfare12, 705-708

Treichler FR, Van Tilburg D (1996) Concurrent Conditional Discrimination Tests of Transitive Inference by Macaque Monkeys: List Linking. J Exp Psychol Anim Behav Process 22, 105-117

Tyler SJ (1972) The behaviour and social organisation of the new Forest ponies. Anim Behav Monogr $5,85-196$

White DJ (2004) Influences of social learning on mate-choice decisions. Learn Behav 32, 105-113 